\title{
Increasing prevalence of extended-spectrum-beta- \\ lactamase among Gram-negative bacilli in Latin America - 2008 update from the Study for Monitoring Antimicrobial Resistance Trends (SMART)
}

Authors

Maria Virginia Villegas ${ }^{1}$ Manuel Guzmán Blanco Jose Sifuentes-Osornio ${ }^{3}$ Flávia Rossi

'MD, MSc, Infectious Diseases - International Center for Medical Research and Training (CIDEIM), Cali, Colombia ${ }^{2} \mathrm{MD}$, Infectious Diseases Hospital Vargas de Caracas, Centro Médico de Caracas,

Caracas, Venezuel

${ }^{3} \mathrm{MD}$, Infectious Diseases

National Institute of

Medical Sciences and

Nutrition Salvador Zubiran,

Mexico City, Mexico

${ }^{4} \mathrm{MD}, \mathrm{PhD}$ - Hospital das

Clínicas da Faculdade de

Medicina de São Paulo, São

Paulo, Brasil

Submitted on: $05 / 14 / 2010$ Approved on: 06/18/2010

Correspondence to:

Maria Virginia Villegas

Cra 125 \#

19-225, Cali, Colombia

Phone: 57-2-5552164

mariavirginia.villegas@

gmail.com

Financial Support: Funding for SMART was provided by Merck, Sharp, \& Dohme.

Conflicts of interest: MVV has received funding from Merck, Sharp and

Dohme; Astra Zeneca;

Pfizer; Bayer, Janssen and

Novartis.

MGB has served on advisory boards for Pfizer; Wyeth; Merck, Sharp \& Dohme; and Biomerieuex. He has received research grants from International Health Management Associates, Inc; Janssen; and Wyeth.

JSO has no conflicts of interest.

FR was an Advisory Board Member for Merck.

\begin{abstract}
Objectives: This analysis of the Study for Monitoring Antimicrobial Resistance Trends (SMART) evaluated the susceptibility patterns of Enterobacteriaceae in Latin America in 2008, with emphasis on susceptibility trends of E. coli and K. pneumoniae. Methods: Clinical isolates were recovered from intra-abdominal infections (IAI) from 23 centers in 10 Latin American countries. Isolates were sent to a central laboratory for confirmation of identification, antimicrobial susceptibility and ESBL testing, following the Clinical Laboratory Standards Institute (CLSI) guidelines. Results: Of 1,003 Gram-negative bacilli collected from intra-abdominal infections, E. coli and $K$. pneumoniae were the most commonly isolated organisms, and $26.8 \%$ of E. coli and $37.7 \%$ of K. pneumoniae were ESBL positive. Ertapenem and imipenem were the most consistently active agents tested; $99 \%$ of ESBLpositive E. coli isolates were susceptible to ertapenem and 100\% to imipenem as well, and $91 \%$ of ESBL-positive K. pneumoniae were susceptible to ertapenem and 98\% to imipenem. Quinolones and cephalosporins were less active, achieving $1.5 \%$ to $76 \%$ inhibition against ESBL-producing E. coli and $3.5 \%$ to $61 \%$ inhibition against $K$. pneumoniae. Conclusions: Local and unit-specific surveillance data is particularly important for selection of empiric therapy and in community-acquired infections as they can help the clinician with antibiotic selection by providing guidance regarding the likely pathogens and their resistance profiles. Our data also confirm the increasing frequency with which ESBL-producing organisms are found in the community setting, with $31.4 \%$ of communityacquired and $24.9 \%$ of hospital-acquired infections found to produce ESBLs. Imipenem and ertapenem are the most active agents tested for ESBL-positive E. coli and K. pneumoniae.
\end{abstract}

Keywords: carbapenems; beta-lactamases; Gram-negative bacteria; drug resistance; ESBL.

[Braz J Infect Dis 2011;15(1):34-39]@Elsevier Editora Ltda.

\section{INTRODUCTION}

Enterobacteriaceae are the most common Gram-negative organisms responsible for intraabdominal infection (IAI) and Escherichia coli is the most frequent pathogen associated with IAI. ${ }^{1}$ While the prevalence of E. coli in diseases such as IAI has remained relatively constant, its overall susceptibility to antibiotics has decreased, as the incidence of extended-spectrum beta-lactamase (ESBL) producing isolates has increased. ESBL-producing Klebsiella pneumoniae has also become a major treatment problem in IAI. ${ }^{2,3}$ Antibiotic resistant ESBL-producing K. pneumoniae has been identified as threats to treatment in Latin America, with resistance observed in both community- and hospitalacquired infections. ${ }^{6}$ In this era of widespread resistance among both community and nosocomial pathogens, improved knowledge of local and regional epidemiology and susceptibility patterns is crucial in order to optimize empiric antibiotic treatment strategies. ${ }^{4,5}$

The Study for Monitoring Antimicrobial Resistance Trends (SMART) is a surveillance study that monitors global susceptibility patterns of Gram-negative bacteria (GNB) from patients hospitalized with IAI. ${ }^{1,7-9}$ The SMART program monitors the activity of amikacin, ampicillinsulbactam, cefepime, cefotaxime, cefoxitin, ceftazidime, ceftriaxone, ciprofloxacin, ertapenem, imipenem, levofloxacin, and piperacillintazobactam against GNB from IAI. Initiated in 2002, there were 120 participating SMART centers worldwide in 2008, with 23 centers in Latin America. This sub-analysis of SMART data evaluated current susceptibility patterns of Enterobacteriaceae recovered from IAI in patients from Latin America in 2008. 


\section{METHODS}

\section{Study sites}

In 2008 there were 23 centers in 10 Latin American countries that participated in SMART. These centers were located in Argentina (2), Brazil (5), Chile (2), Colombia (3), Dominican Republic (1), Guatemala (1), Mexico (3), Panama (1), Peru (2), and Venezuela (3).

\section{Isolate collection}

Up to 100 consecutive non-duplicate clinical isolates were collected prospectively from patients with IAI at each center. Only the first isolate of a particular species from any patient could be included for the entire collection period. Gramnegative aerobic and facultative bacteria were cultured from IAI sites such as appendix, peritoneum, colon, bile, pelvis and pancreas. Isolates were obtained during surgery, or through paracentesis or percutaneous aspiration of abscesses. Isolates from blood, urine, stool, abdominal drains or drainage bottles, superficial wounds, and perirectal abscesses were excluded. Multiple organisms obtained from one specimen were acceptable provided each was a unique initial GNB. Isolates collected within $<48$ hours of hospitalization were categorized as community-acquired and those collected $>48$ hours after hospitalization were categorized as nosocomial.

\section{Antimicrobial susceptibility testing}

Isolates were sent to a central laboratory (Laboratories International for Microbiology Studies, a subsidiary of International Health Management Associates, Inc., Schaumburg, Illinois, USA) for confirmation of identification and antimicrobial susceptibility testing. Susceptibility testing was performed following Clinical Laboratory Standards Institute (CLSI) guidelines ${ }^{10}$ using dehydrated broth microdilution panels prepared by MicroScan (Siemens Medical Solutions Diagnostics, West Sacramento, California, USA). ESBL testing was done according to CLSI guidelines (CLSI 2009), with a positive test requiring at least three doubling dilution decrease in minimum inhibitory concentration of ceftazidime or cefotaxime in the presence of clavulanic acid. Quality control testing was done following CLSI ${ }^{11}$ and manufacturer (MicroScan) guidelines, using reference strains E. coli ATCC 25923, E. coli ATCC 35218, Pseudomonas aeruginosa ATCC 27853, and K. pneumoniae ATCC 700603. The following antimicrobials, obtained from the panel manufacturer, were tested: amikacin, ampicillin-sulbactam, cefepime, cefotaxime, cefoxitin, ceftazidime, ceftriaxone, ciprofloxacin, ertapenem, imipenem, levofloxacin and piperacillin-tazobactam.

\section{RESULTS}

Of the 1,003 GNB isolates collected, 92\% were represented by 9 species: E. coli $(\mathrm{n}=504), K$. pneumoniae $(\mathrm{n}=151)$, Enterobacter cloacae $(\mathrm{n}=69)$, . aeruginosa $(\mathrm{n}=68)$,
Proteus mirabilis $(\mathrm{n}=37)$, Citrobacter freundii $(\mathrm{n}=24)$, Acinetobacter baumanii $(\mathrm{n}=24)$, Serratia marcescens $(\mathrm{n}=23)$, and Enterobacter aerogenes $(\mathrm{n}=23)$. Twenty-two other species comprised the remaining $8 \%$ of isolates. The most commonly isolated organism was E. coli, of which 135 of the isolates (26.8\%) were ESBL positive. Among the K. pneumoniae isolates, 57 (37.7\%) were ESBL positive. Table 1 shows the proportion of ESBL-producing isolates in Latin America. Data for the overall Latin American region are described here, with less emphasis on country-specific data, because the number of isolates collected from each country varied and was in some cases too small to be able to discern trends. For example, 86 E. coli were collected in Argentina, 83 in Chile, 79 in Venezuela, 61 in Guatemala, 43 in Mexico and in Panama, 41 in Brazil, 38 in Colombia, 26 in Puerto Rico, and 4 in Dominican Republic.

Antimicrobial susceptibilities of the most commonly isolated pathogens ( $>50$ isolates) are summarized in Table 2. Susceptibilities of non-ESBL-producing E. coli, Klebsiella, and Proteus isolates to the carbapenems ranged from $96.8 \%$ to $100 \%$ (ertapenem) and $98.9 \%$ to $100 \%$ (imipenem). The antimicrobial susceptibilities of the ESBL-producing organisms E. coli, K. pneumoniae, and K. oxytoca are shown in Figure 1. Among the antimicrobials tested, ertapenem and imipenem remained the most consistently active against all pathogens, with $99.3 \%$ of ESBL-producing E. coli and 91.2\% of ESBL-producing K. pneumoniae susceptible to ertapenem and $100 \%$ of ESBL-producing E. coli and $98.2 \%$ of ESBL-producing $K$. pneumoniae susceptible to imipenem. All $P$. mirabilis isolates were susceptible to ertapenem and imipenem. Susceptibilities were lower for other agents, particularly against ESBL-producing strains. In particular, ESBL producers appeared to have reduced susceptibility to commonly-used antibiotics including cephalosporins, fluoroquinolones, and ampicillin-sulbactam. The susceptibility of ESBL-positive E. coli and K. pneumoniae varied somewhat by country, although they were most consistently susceptible to ertapenem and imipenem and least likely to be susceptible to cefepime or ciprofloxacin. Too few isolates were collected to identify definitive trends, however. It is worth noting that ESBL-producing E. coli (97.8\%) and K.pneumoniae $(100 \%)$ were uniformly resistant to ampicillin-sulbactam; nonESBL-producing E. coli (51.5\%) and K. pneumoniae (40.4\%) had reduced susceptibility to ampicillin-sulbactam.

Table 1. Frequency of ESBL-positive and ESBL-negative E. coli and Klebsiella spp. in 2008 in the Latin American region

\begin{tabular}{lcccc}
\hline \multirow{2}{*}{ Pathogen } & \multicolumn{2}{c}{ ESBL +} & \multicolumn{2}{c}{ ESBL - } \\
& $\mathbf{n}$ & $\mathbf{\%}$ & $\mathbf{n}$ & \% \\
\hline E. coli & 135 & 26.8 & 369 & 73.2 \\
\hline K. oxytoca & 4 & 20 & 16 & 80 \\
\hline K. pneumoniae & 57 & 37.7 & 94 & 62.3 \\
\hline
\end{tabular}




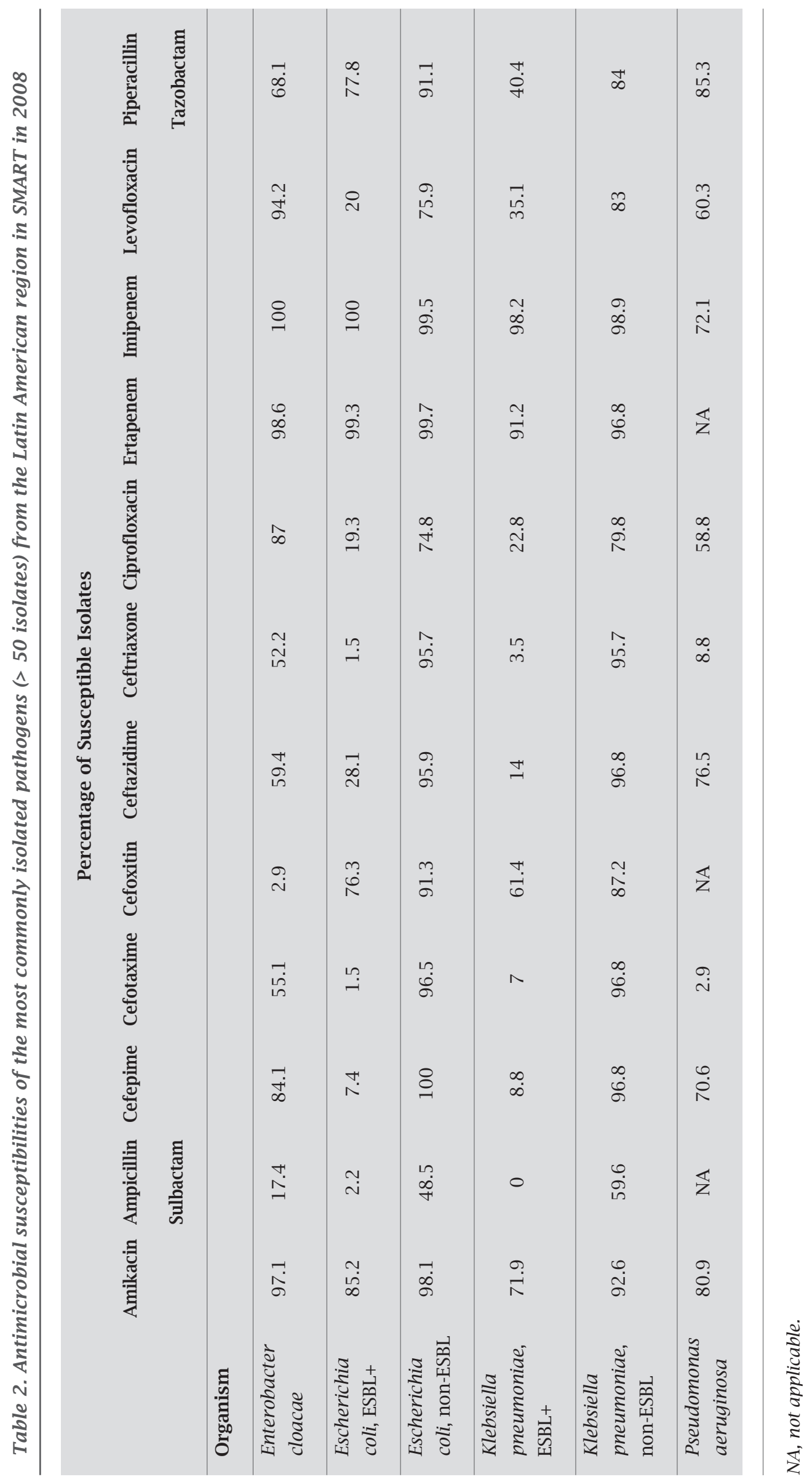


The frequency of ESBL-positive E. coli and Klebsiella spp. from community-acquired and hospital-acquired infections is shown in Table 3. The rate of ESBL-producing infections in the community-acquired group was $28.7 \%$; the rate of ESBL-positive strains implicated in hospital-acquired infections was slightly lower (24.4\%).Overall, $99 \%$ of community-acquired ESBL-producing E. coli were susceptible to ertapenem,

Figure 1. Antimicrobial susceptibilities of ESBL-producing E. coli and K. pneumoniae in Latin America (2002-2008). Susceptibilities are based on in vitro minimum inhibitory concentration data.
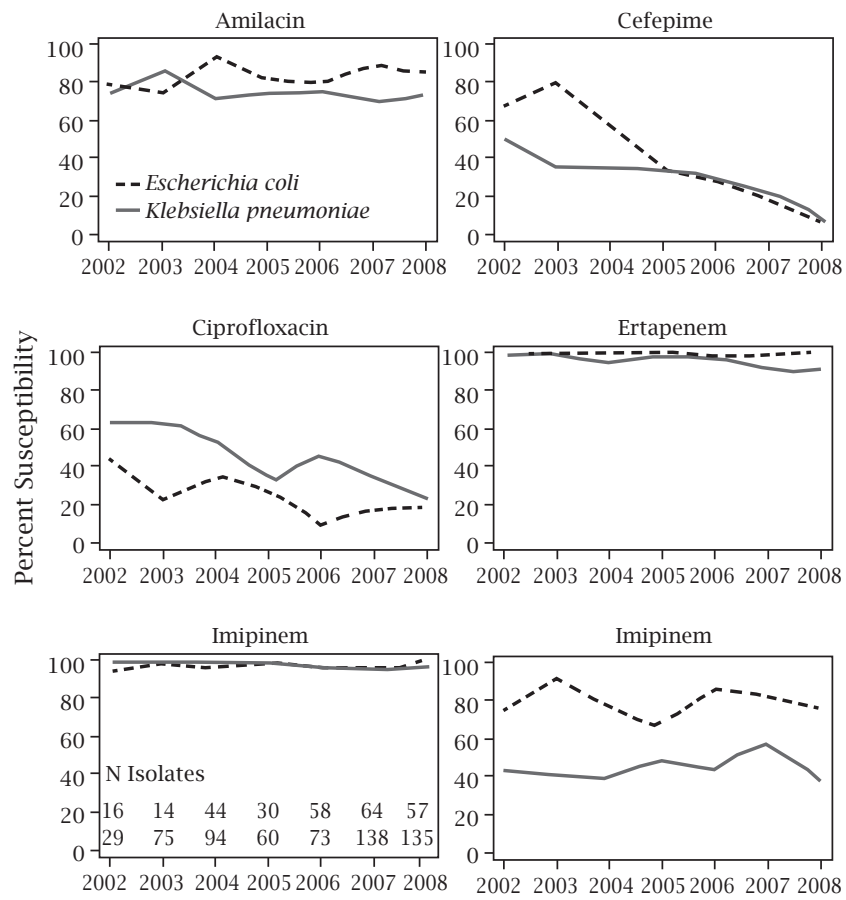

Year Collected

Table 3. Frequency of ESBL-positive and ESBL-negative E. coli and Klebsiella spp. in community-acquired and hospital-acquired infections in Latin America

\begin{tabular}{|lcccc|}
\hline \multirow{2}{*}{ Infection Type $^{\mathbf{a}}$} & \multicolumn{2}{c}{ ESBL + } & \multicolumn{2}{c|}{ ESBL - } \\
& $\mathbf{n}$ & $\mathbf{\%}$ & $\mathbf{n}$ & $\%$ \\
\hline $\begin{array}{l}\text { Community-acquired } \\
\text { infection }\end{array}$ & 82 & 26.7 & 204 & 71.3 \\
\hline $\begin{array}{l}\text { Hospital-acquired } \\
\text { infection }\end{array}$ & 52 & 24.4 & 161 & 75.6 \\
\hline Not specified & 1 & 20 & 4 & 80 \\
\hline Total & $\mathbf{1 3 5}$ & $\mathbf{2 6 . 8}$ & $\mathbf{3 6 9}$ & $\mathbf{7 3 . 2}$ \\
\hline
\end{tabular}

${ }^{a}$ Community-acquired infection indicates that the isolate was collected within $<48$ hours of hospitalization; hospitalacquired infection indicates that the isolate was collected $>48$ hours following hospitalization.
$100 \%$ to imipenem, $88 \%$ to amikacin, $6 \%$ to cefepime, $22 \%$ to ciprofloxacin, and $73 \%$ to piperacillin/tazobactam. With hospital-acquired infections, 99\% of ESBL-producing $E$. coli were susceptible to ertapenem, $100 \%$ to imipenem, $85 \%$ to piperacillin/tazobactam, $81 \%$ to amikacin, $15 \%$ to ciprofloxacin, $10 \%$ to cefepime, and only $2 \%$ to ampicillinsulbactam. Similarly, $92 \%$ and $93 \%$ of community- and hospital-acquired ESBL-producing $K$. pneumoniae were respectively susceptible to ertapenem and $98 \%$ and $100 \%$ to imipenem, while $74 \%$ and $67 \%$ of community- and hospital-acquired ESBL-producing K. pneumoniae were susceptible to amikacin, $12 \%$ and $0 \%$ to cefepime, $19 \%$ and $33 \%$ to ciprofloxacin, and $41 \%$ and $50 \%$ to piperacillin/ tazobactam, respectively.

\section{DISCUSSION}

The frequency of ESBL-producing E. coli and Klebsiella in Latin America was generally higher in 2008 compared with that reported from SMART in previous years. Overall, $26 \%$ of E. coli and 35\% of K. pneumoniae isolated from IAI in the Latin American region produced ESBLs, compared with $10 \%$ and $14 \%$ of E. coli and K. pneumoniae from SMART in 2003 , and $10 \%$ and $18 \%$ in $2004 .{ }^{1,7}$ Other surveillance studies have also noted relatively high rates of ESBL-producing pathogens in Latin America. SENTRY results from Latin America (1997-1998) indicated that $46.9 \%$ of K. pneumoniae strains $(\mathrm{n}=1,225)$ produced ESBLs, with rates ranging from $26.2 \%$ in Venezuela to $52 \%$ in Mexico. ${ }^{12}$ The Tigecycline Evaluation and Surveillance Trial (TEST) reported rates of ESBL production of $13.5 \%$ among E. coli and $44 \%$ among K. pneumoniae isolates in Latin America (2004-2006). ${ }^{13}$ A higher percentage of ESBL-producing Enterobacteriaceae were observed in South America than in North America according to the Meropenem Yearly Susceptibility Test Information Collection (MYSTIC) surveillance study between 1997 and 2003. ${ }^{14}$ While $18.1 \%$ of E. coli from South America were ESBL positive, only $7.5 \%$ of isolates from North America were ESBL positive; $51.9 \%$ of $K$. pneumoniae from South America were ESBL producers versus $12.3 \%$ of North American strains. ${ }^{13}$ In Brazil, 24\% of 498 Enterobacteriaceae analyzed from 2002 to 2003 were ESBL producers, including $57 \%$ of the K. pneumoniae. ${ }^{15} \mathrm{~A}$ similar study in Colombia in 2002 examined 1,074 E. coli and 394 K. pneumoniae clini$\mathrm{cal}$ isolates and found a high prevalence of ESBLs in most hospitals, among both E. coli (11.8\%) and K. pneumoniae (32.6\%). ${ }^{16}$ These rates were similar to rates reported in other countries in Latin America; some of the hospitals participating in this study, however, had rates that were substantially higher, up to $71.4 \%$ for K. pneumoniae and $16.7 \%$ for E. coli in some intensive care units. ${ }^{16}$ The rates of ESBL production in Latin America appear to be approaching those of the Asia/Pacific region, where ESBL frequencies of $40 \%$ were observed in SMART. ${ }^{17}$ 
Our data also confirm the increasing frequency with which ESBL-producing organisms are found in the community setting, with $31.4 \%$ of community-acquired and $24.9 \%$ of hospital-acquired infections found to produce ESBLs. The community-acquired rate is similar to that reported in the Asia/Pacific region (28\%) although the rate of hospital-acquired ESBL infection was much higher in the Asia/Pacific region (55.4\%). ${ }^{17}$ In SMART, isolates collected within $<48$ hours of hospitalization were categorized as community-acquired and those collected $>48$ hours after hospitalization were categorized as nosocomial, thus it is possible that some cases were miscategorized using these criteria.

Cephalosporins and quinolones susceptibilities of ESBLproducing organisms in Latin America declined from 2002 to 2008 , while these organisms remained consistently susceptible to the carbapenems and, for the most part, to amikacin. SENTRY data based on nearly 20,000 community and hospital clinical isolates collected in Latin America between 1997 and 2001, indicated that ESBL-producing E. coli and $K$. pneumoniae remained uniformly susceptible to imipenem and meropenem, with high-level resistance to cephalosporins (e.g., $48.4 \%$ cefepime resistance among $K$. pneumoniae), fluoroquinolones (e.g., 49.2\% levofloxacin resistance among E. coli) and, in some cases, to beta-lactams such as piperacillin/ tazobactam (55.6\% resistance among E. coli and 34.3\% resistance among $K$. pneumoniae).$^{18}$ Imipenem was the most active agent against both ESBL-producing E. coli and K. pneumoniae in SENTRY and TEST. ${ }^{13,18}$ Overall susceptibility of ESBLproducing Klebsiella species reported by SENTRY for 1997 to 2002 showed resistance rates from $35.8 \%$ to $46.9 \%$, although rates of ESBL-producing Klebsiella were lower in 2001 to 2002 ( $35.8 \%$ to $39.5 \%$ ) compared to 1997 to 2000 (43.5 to $46.9 \%$ ), highlighting yearly variations. ${ }^{19}$ While ampicillin-sulbactam is still commonly used in some Latin American countries, both ESBL-producing and non-ESBL-producing E. coli and K. pneumoniae were highly resistant. For all of the results in this study, it is important to note that susceptibility is defined in terms of minimum inhibitory concentrations and there is no clinical correlation with treatment.

Physicians have relied on the ability of antibiotics to treat infections for many decades. With high-level antibiotic resistance manifesting around the world, patients are more likely to be treated with inadequate antibiotic therapy and consequently more likely to die from infections and infectious complications. ${ }^{20-22}$ The selection of antibiotic resistant strains due to inappropriate antibiotic use and overuse has had a negative impact on hospital ecology. ${ }^{23}$ For example, a retrospective case-control study that included all cases of $K$. pneumoniae bacteremia from a single center in Mexico (1993-2002) reported that an ESBL-producing isolate was found in $17 / 121$ cases (14\%), and that prior cephalosporin use $(\mathrm{p}=0.039)$ and previous stay in the intensive care unit ( $p=0.033$ ) were significant risk factors for infection with ESBLproducing K. pneumoniae. $^{24}$
Local and unit-specific surveillance data can inform antibiotic selection by providing guidance regarding the likely pathogens and their resistance profiles. This is particularly important for selection of empiric therapy and in community-acquired infections where microbiologic data and antibiotic use information may not be available. A call for a Latin American surveillance network was made a decade ago to address these concerns. ${ }^{6}$ The SMART database provides accessible longitudinal local, regional, and worldwide data on the susceptibility of isolates from IAI, with the limitations that sites are not uniformly distributed and the number of isolates collected at each site is variable. Nevertheless, local monitoring of ESBL producer prevalence and the susceptibility of these pathogens to commonly-used antibiotics is needed to improve patient outcomes and preserve the efficacy of the available antibiotic agents.

\section{ACKNOWLEDGMENTS}

The authors wish to acknowledge Wendy Horn, Ph.D. of Insight Communication for editorial and writing assistance and Robert Balshaw, Ph.D. of SyreonCorporation for analytical assistance, which were funded by Merck, Sharp \& Dohme.

\section{REFERENCES}

1. Rossi F, Baquero F, Hsueh P-R et al. In vitro susceptibilities of aerobic and facultative Gram-negative bacilli isolated from patients with intra-abdominal infections worldwide: 2004 Study for Monitoring Antimicrobial Resistance Trends (SMART). J Antimicrob Chemother. 2006; 58:205-210.

2. Falagas ME, Karageorgopoulos DE. Extended-spectrum betalactamase-producing organisms. J Hosp Infect. 2009; 73:345-354.

3. Paterson DL. Resistance in Gram-negative bacteria: Enterobacteriaceae. Am J Infect Control 2006; 34:S20-8.

4. Carmeli Y. Strategies for managing today's infections. Clin Microbiol Infect. 2008; 14(Suppl. 3):22-31.

5. Green D. Selection of an empiric antibiotic regimen for hospital-acquired pneumonia using a unit and culture-type specific antibiogram. J Intensive Care Med. 2005; 20:296-301.

6. Villegas MV, Kattán JN, Quinteros MG, Casellas JM. Prevalence of extended spectrum beta-lactamases (ESBLs) in Europe and in the rest of the world: differences and similarities with South America. Clin Microbiol Infect. 2008; 14(Suppl. 1):154-158.

7. Paterson D, Rossi F, Baquero F et al. In vitro susceptibilities of aerobic and facultative Gram-negative bacilli isolated from patients with intra-abdominal infections worldwide: the 2003 Study for Monitoring Antimicrobial Resistance Trends (SMART). J Antimicrob Chemother. 2005; 55:965-973.

8. Chow J, Satishchandran V, Snyder TA et al. In vitro susceptibilities of aerobic and facultative Gram-negative bacilli isolated from patients with intra-abdominal infections worldwide: the 2002 Study for Monitoring Antimicrobial Resistance Trends (SMART). Surg Infect. 2006; 6:439-448.

9. Badal, R, Bouchillon S, Johnson A, Hackel M, Hoban, D. Global susceptibility patterns of E. coli from intra-abdominal infections to ertapenem and comparators-SMART 2008. 49th Interscience Conference on Antimicrobial Agents and Chemotherapy, September 12-15, 2009, San Francisco, CA, USA. 
10. Clinical and Laboratory Standards Institute, 2008. Methods for Dilution Antimicrobial Susceptibility Tests for Bacteria That Grow Aerobically; Approved Standard-Seventh Edition, in Document M7-A7. Clinical and Laboratory Standards Institute (CLSI), Wayne, PA 19087-1898 USA.

11. Clinical and Laboratory Standards Institute, 2009. Performance Standards for Antimicrobial Susceptibility Testing; Fourteenth Informational Supplement. CLSI document M100-S19. Clinical and Laboratory Standards Institute (CLSI), Wayne, PA 19087-1898 USA.

12. Sader H, Gales A, Granacher T. Pfaller M, Jones R, and the SENTRY Study Group (Latin America). Prevalence of antimicrobial resistance among respiratory tract isolates in Latin America: results from SENTRY antimicrobial surveillance program (1997-98). Braz J Infect Dis. 2000; 4:245-253.

13. Reinert RR, Low DE, Rossi F, Zhang X, Wattal C, Dowzicky MJ. Antimicrobial susceptibility among organisms from the Asia/ Pacific Rim, Europe and Latin and North America collected as part of TEST and the in vitro activity of tigecycline. J Antimicrobial Chemother. 2007; 60:1018-1029.

14. Turner PJ. (2005). Extended-Spectrum Beta-Lactamases. Clin Infect Dis. 2005; 41(Suppl. 4):S273-S275.

15. Nogueira Kda. Sm, Higuti IH, do Nascimento AJ et al. Occurrence of extended-spectrum beta-lactamases in Enterobacteriaceae isolated from hospitalized patients in Curitiba, southern Brazil. Braz J Infect Dis. 2006; 10:390-395.

16. Villegas MV, Correa A, Perez F, Zuluaga T, Quinn JP. Prevalence and characterization of ESBLs from E. coli and K. pneumoniae isolates in Colombian hospitals. Diag Micro Infect Dis. 2004; 49:217-222.

17. Hawser SP, Bouchillon SK, Hoban DJ et al. Emergence of high levels of extended-spectrum-b-lactamase-producing gramnegative bacilli in the Asia-Pacific Region: Data from the study for monitoring Antimicrobial Resistance Trends (SMART) program, 2007. Antimicrob Agents Chemother. 2009;53:3280-3284.
18. Sader HS, Jones RN, Gales AC, Silva JB, Pignatari1 AC and the SENTRY Participants Group (Latin America). SENTRY antimicrobial surveillance program report: Latin American and Brazilian results for 1997 through. Braz J Infect Dis. 2004; 8:25-79.

19. Biedenbach DJ, Moet GJ, Jones RN. Occurrence and antimicrobial resistance pattern comparisons among bloodstream infection isolates from the SENTRY Antimicrobial Surveillance Program (1997-2002). Diagn Microbiol Infect Dis. 2004; 50:59-69.

20. Peña C, Gudiol C, Calatayud L et al. Infections due to Escherichia coli producing extended-spectrum beta-lactamase among hospitalised patients: factors influencing mortality. J Hosp Infect. 2008; 68:116-122.

21. Tumbarello M, Sali M, Trecarichi EM et al. Bloodstream infections caused by extended-spectrum-beta-lactamaseproducing Escherichia coli: Risk factors for inadequate initial antimicrobial therapy. Antimicrob Agents Chemother. 2008; 52:3244-3252.

22. Tumbarello M, Sanguinetti M, Montouori E et al. Predictors of mortality in patients with bloodstream infections caused by extended-spectrum-beta-lactamase-producing Enterobacteriaceae: Importance of initial antimicrobial therapy. Antimicrob Agents Chemother. 2008; 53:1987-1994.

23. Paterson DL. "Collateral damage" from cephalosporin or quinolone antibiotic therapy. Clin Infect Dis. 2004; 38(Suppl. 4):S341-S345.

24. Mosqueda-Gómez JL, Montaño-Loza A, Rolón AL et al. Molecular epidemiology and risk factors of bloodstream infections caused by extended-spectrum beta-lactamaseproducing Klebsiella pneumoniae: A case-control study. Int J Infect Dis. 2008; 12:653-659. 